\section{Técnica de mock up y estratificación por capas anatómicas}

\author{
Mock up technique and anatomical caps stratification
}

\begin{abstract}
Resumen
Las exigencias estéticas por parte de los pacientes son cada vez mayores y esto demanda al profesional mayor preparación para ofrecer la mejor alternativa de tratamiento. Los materiales para uso odontológico, así como las técnicas restauradoras, han evolucionado muy rápidamente. Las resinas compuestas nos dan una alternativa más de tratamiento en el sector anterior ya que cuentan con buenas propiedades tanto estéticas como mecánicas. Con la evolución de las resinas compuestas podemos lograr mucha naturalidad haciendo restauraciones cada vez mejores. En el presente artículo se detalla la técnica de Mock Up y la de Reconstrucción por Estratos Anatómicos en la resolución de un caso clínico. El objetivo fue devolver la estética y la función al sector anterosuperior. Los resultados fueron satisfactorios para el paciente consiguiendo así la naturalidad y recuperación de la función perdida en el sector anterior. Se puede concluir que el conocimiento de los materiales restauradores así como de las diversas técnicas son fundamentales para realizar una adecuada restauración.

Palabras clave: resinas compuestas, restauraciones anteriores, normas estéticas
\end{abstract}

\section{Abstract}

Patient's aesthetic requirements are increasing and this demands the greatest professional preparation to provide the best treatment option. The materials for use in dentistry and restorative techniques have evolved very quickly. Composite resins give us an alternative treatment in the anterior sector because they have good aesthetic and mechanical properties. With the evolution of the composite resins we can achieve very natural restorations doing them better and better. This article details Mock Up's technique and reconstruction by anatomical stratification in the resolution of a case. The aim was to restore aesthetics and function to anterior sector. The results were satisfactory for the patient obtaining this way the naturalness and recovery of lost function in the anterior sector. It can be concluded that the knowledge of restorative materials as well as various techniques are essential for a proper restoration.

Key works: composite resins, anterior restorations, aesthetic standards.
Casos Clínicos

\section{César Lamas Lara', Giselle Angulo de la Vega ${ }^{2}$}

Cirujano Dentista, Alumno del Diplomado de Odontología Restauradora y Estética de la Facultad de Odontología de la UNMSM.

2 Cirujano Dentista, Alumna de la Especialidad de Rehabilitación Oral de la Facultad de Odontología de la UNMSM.

Correspondencia:

CD César Lamas Lara

Facultad de Odontología. UNMSM. Av. Germán Amézaga s/n. Lima 1. Perú

Teléf.: 999-491-403

E-mail: cesar2579@hotmail.com

Fecha de recepción: 11-02-11

Fecha de aceptación: 15-04-11

\section{Introducción}

Restaurar piezas dentarias en el sector antero superior exige por parte del profesional un conocimiento a cabalidad de los materiales, así como de la técnica a emplear, ya que este sector representa un factor muy importante en la estética del rostro.

Conocer las características anatómicas y funcionales de las piezas en el sector anterior es de vital importancia para realizar restauraciones duraderas. No basta con realizar una restauración solamente estética si esta no cumple con los requisitos de función en nuestro sistema estomatognático. ${ }^{1}$

Antes de realizar cualquier procedimiento resturador debemos considerar los posibles problemas estéticos y de acuerdo a la situación elegir el tipo de material más idóneo para cada caso. ${ }^{2}$

En la actualidad hay muy buenos materiales restauradores, pero lo más importante es el conocimiento profundo de los mismos y una técnica adecuada. De nada nos sirve utilizar el que creemos el mejor sistema restaurador si no lo dominamos a cabalidad ya que cada material exige una previa preparación para que nos brinde resultados satisfactorios.

Las restauraciones en el sector anterior requieren por parte del profesional conocimientos tanto teóricos, científicos y artísticos, así como habilidad manual con el fin de reproducir los detalles anatómicos. ${ }^{3}$ y 4

Las resinas compuestas como material restaurador directo cumplen con los requisitos de simplificación de los diseños cavitarios, preservación de la estructura dentaria, simplicidad de la técnica a aplicar, multiplicidad de matices y viscosidades; y sobretodo proveer una estética bastante adecuada para restauraciones tanto anteriores como posteriores 5,6 y 7 .

Actualmente existe una demanda de estética dental por parte de los pacientes que obliga al profesional a mejorar su preparación, los niveles de análisis, comunicación y presentación de sus procedimientos restauradores. ${ }^{8}$

La técnica de Mock Up consiste en aplicar resina compuesta directamente sobre la estructura dentaria a restaurar con el fin de simular de un manera más precisa las características que las piezas dentarias han perdido y de esta manera obtener un patrón que sirva para la posterior restauración definitiva.

La estratificación por capas anatómicas consiste en la aplicación sucesiva de incrementos de resina compuesta de tal manera que logre mimetizar lo mejor posible a las piezas dentarias; así se utilizan diferentes opacidades de resina compuesta para simular los diferentes tejidos dentarios para obtener resultados más naturales. Esta técnica fue descrita por Vanini, Baratieri y Dietschi. ${ }^{9-12}$

El objetivo del caso clínico fue devolver la estética y la función del sector anterosuperior realizando procedimientos conservadores al aplicar resinas compuestas. 


\section{Reporte del caso}

Paciente de sexo femenino de 20 años de edad que acude a la Clínica de la Facultad de Odontología de la Universidad Nacional Mayor de San Marcos presentando restauraciones defectuosas en el sector anterior preocupada por su problema estético.

La paciente refiere antecedentes de fracturas de las coronas en las piezas $11 \mathrm{y}$ 21 hace 10 ańos y reconstrucción con resina compuesta.

$\mathrm{Al}$ examinar el sector anterosuperior se puede apreciar restauraciones defectuosas con resina compuesta y presencia de lesiones de caries recidivante a nivel de las piezas 1.1 y 2.1 (Fig. 1). Paciente con buen estado de salud general, sin riesgo sistémico al tratamiento estomatológico.

Se realizó pruebas de sensibilidad pulpar para determinar la vitalidad de las piezas 11 y 21. y un análisis de las piezas comprometidas encontrando una inclinación de la línea media dentaria, diferencias entre los anchos de los dos centrales de $0.5 \mathrm{~mm}$., contactos interdentales desiguales, líneas visibles de los incrementos de resina aplicadas anteriormente además de la presencia de caries recidivante (Fig. 2).

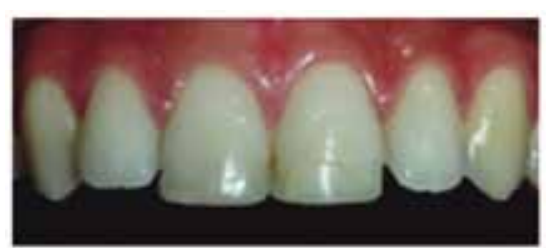

Fig. 1. Vista Vestibular.

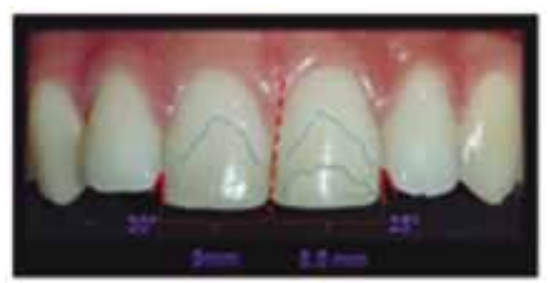

Fig. 2. Esquematización de las diferencias entre las restauraciones de las piezas 11 y 21 .

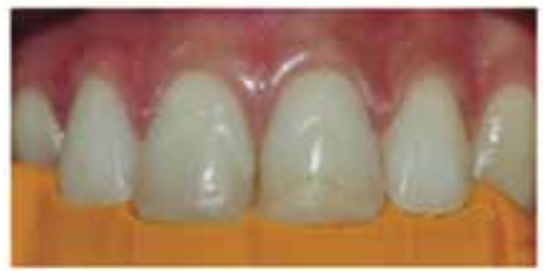

Fig.3: Confección de una guía de silicona directamente en boca.

\section{Procedimiento clínico}

Se decidió aplicar incrementos de resina compuesta sobre las restauraciones anteriores sin la colocación de ácido y de adhesivo (técnica de Mock Up) con el fin de mejorar los anchos de las piezas comprometidas así como los ángulos de contacto interdental. Una vez obtenidas las características deseadas, se confeccionó una guía de silicona con masa pesada de las superficies palatinas directamente en boca (Fig. 3). Con aislamiento absoluto del sector anterosuperior; se realizó la remoción de las restauraciones antiguas y la eliminación del tejido cariado; se pulieron las superficies comprometidas y se biseló la preparación a nivel vestibular. Se aplicó ácido ortofosfórico al $37 \%$ sobre las superficies preparadas, 15 segundos en dentina y 30 segundos sobre esmalte; se lavó con abundante agua y se secaron las superficies con papel absorbente sin resecar las superficies. Se aplicó dos capas de un adhesivo de quinta generación (Fig. 4) y se frotó hasta evaporar el solvente del adhesivo, posteriormente se fotocuró por 20 segundos. Se colocó la guía de silicona confeccionada en boca y se procedió a la estratificación por capas anatómicas aplicando una capa de resina translucida que nos servirá como esmalte palatino (Fig. 5), se fotocuró el incremento de resina compuesta por 20 segundos. Posteriormente se aplicó una resina opaca de color A2 para reemplazar a la dentina y en el borde incisal colocamos una resina de cuerpo de color A1 para dar el efecto de línea alba del borde incisal (Fig.6) y se fotocuró los incrementos de resina compuesta por 20 segundos. Se aplicó un incremento de resina translucida en los espacios dejados al colocar la resina de dentina para dar un mayor efecto

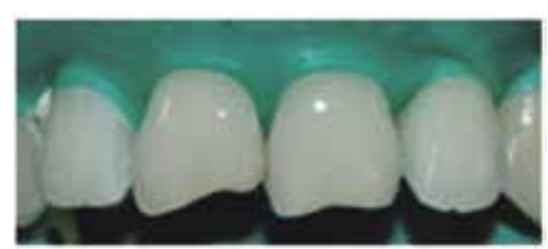

Fig. 4. Aplicación de un adhesivo de quinta generación.

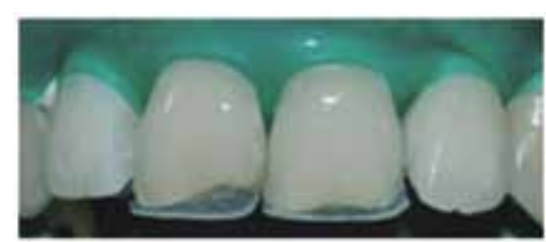

Fig. 5. Aplicación de una resina translucida como esmalte palatino. de translucidez. Se colocó una resina compuesta para esmalte de color A1 que cubra toda la cara vestibular que nos va a simular el esmalte vestibular y se fotocuró por 20 segundos. (Fig. 7). Posteriormente se retiró el aislamiento absoluto y con una fresa multilaminada hacemos los retoques de las restauraciones, controlamos la oclusión y posteriormente se dio el acabado y pulido a las restauraciones (Fig. 8).

\section{Discusión}

Los resultados fueron satisfactorios para la paciente ya que se logró devolver la estética y la función a los incisivos centrales superiores cuyo papel en la estética del rostro es muy importante. Se preservó la mayor cantidad de estructura dentaria sana y se restauró con un procedimiento sencillo y con resultados previsibles, otorgando así a la paciente la naturalidad perdida anteriormente.

Las restauraciones directas con resinas compuestas, ofrecen una alternativa de tratamiento estética y funcional frente a otras restauraciones convencionales, como carillas y coronas cerámicas, pues preservamos mayor cantidad de estructura dentaria, reducimos el número de citas clínicas y costos para el paciente. 13 y 14

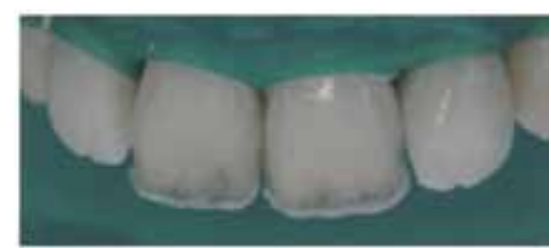

Fig. 6. Aplicación de una resina opaca como dentina color A2 y una resina de color A1 para el efecto de la línea alba incisal.

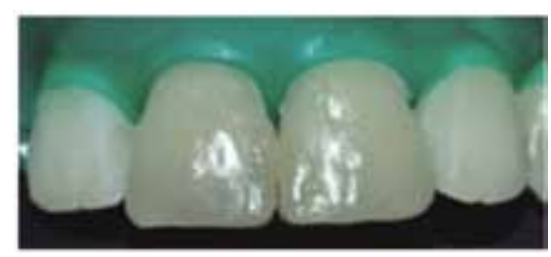

Fig. 7. Aplicación de una resina de esmalte para cubrir toda la cara vestibular.

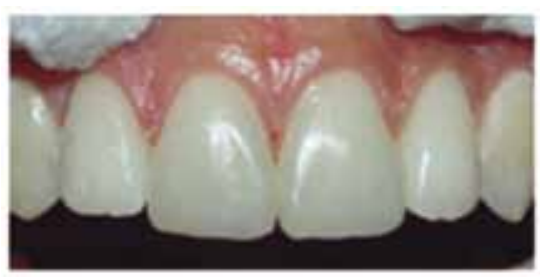

Fig. 8 Restauraciones terminadas 
La elección de la técnica restauradora con resinas compuestas a utilizar dependerá del dominio de la misma y del nivel de complejidad de la situación clínica que se presente. ${ }^{15}$ Utilizando la técnica de estratificación por capas anatómicas en este caso se logró una adecuada mimetización de la restauración a las estructuras dentales remanentes.

\section{Conclusiones}

Las resinas compuestas de uso directo son una muy buena alternativa de tratamiento para los casos de restauraciones anteriores. El profundo conocimiento de los materiales y las técnicas a utilizar van a facilitar la ejecución de un tratamiento satisfactorio. Cabe destacar que no todas las resinas compuestas se comportan de la misma manera, tanto en sus propiedades físicas y mecánicas como en su manipulación. No es suficiente realizar una restauración visualmente correcta si esta no cumple su función dentro del sistema estomatognático.

\section{Referencias bibliográficas}

1. Lamas C; Angulo G; Reconstrucción del Sector Anterior con Resinas Compuestas, Odontol. Sanmarquina, 2009; 12 (2): 90 - 92
2. Nogueira de Sá MP, Pascotto RC. Faceta direta em resina composta com recurso de uma matriz de acetato - relato de caso clínico. Revista dental press de estética. 2004; 1:101111.

3. Medeiros C, Bernal C, Braz da Silva R. Aesthetics Rehabilitation Through Direct Facets - Report of cases. Acta Odontológica 2009; 47(2): $1-14$.

4. Bloom DR, Padayachy J N. Smile lifts - a functional and aesthetic perspective. Br Dent J 2006; 199203.

5. Albers HF. Tooth colored restorations: principles and techniques. 9th edition. BC Decker Publishing; Ontario, Canada. 2002. p. 203

6. Conceição EN et al. Restaurações Estéticas. Compósitos, Cerâmicas e Implantes. Ed Artmed. Porto Alegre, Brasil. 2005. p. 152.

7. Ardu S, Krejci I. Biomimetic direct composite stratification technique for the restoration of anterior teeth. Quintessence Inter 2006; 37(3): 167-74.

8. Guerra CM, Maravankin FR. Estética en odontologia. El análisis estético como factor de decisión. Actas Odontol 2006; III (2): 15-23.
9. Dietschi D. Free-hand composite resin restorations: A Key to anterior aesthetics. Pract Periodont Aesth Dent 1995; 7:15-25.

10. Baratieri LN et al. Estètica. Restauraçóes adesivas diretas em dentes anteriores. Quintessence 1995. pp. $75-77$

11. Vanini L. Light and color in anterior composite restorations. Pract Periodontics Aesthet Dent 1996; 8(7): 673-82

12. Magne P, Holz J. Stratification of composite restorations. Systematic and durable replication of natural aesthetics. Pract Periodont Aesthet Dentistry 1996; 8: 61-8.

13. Ardu S, Krejci I. Biomimetic direct composite stratification technique for the restoration of anterior teeth. Quintessence Inter 2006; 37(3): 167-74.Hidalgo RC. Técnica de estratificación por capas naturales a mano alzada con resinas compuestas. Visión Dental 2008; 11 (3): 412-418

14. Conceição EN et al. Restauraçóes Estéticas. Compósitos, Cerâmicas e Implantes. Porto Alegre: Ed Artmed; 2005. pp. 152-3. 